\title{
UM NOVO MÉTODO PARA AGRUPAR PARÂMETROS QUIMIOTAXONÔMICOS
}

\author{
V. P. Emerenciano, G. V. Rodrigues, S. A. V. Alvarenga e P. A. T. Macari
}

Instituto de Química - Universidade de São Paulo - CP 26.077 - 05599-970 - São Paulo - SP

M. A. C. Kaplan

NPPN - Universidade Federal do Rio de Janeiro - Rio de Janeiro - RJ

Recebido em 19/8/96; aceito em 22/10/97

\begin{abstract}
A NEW METHOD FOR GROUPING CHEMOTAXONOMIC PARAMETERS. This work presents a new methodology for searching the present state of chemical evolution for different taxa, grouping five classes of secondary metabolites. Ocurrences of 10370 natural products isolated from Asteraceae were used to exemplify the method. Relationships among tribes are discussed.
\end{abstract}

Keywords: Asteraceae; sesquiterpene lactones; diterpenes; triterpenes; flavonoids; coumarins; chemotaxonomy.

\section{INTRODUÇÃO}

Durante muitos anos vários autores têm estudado sistematicamente a evolução da família Asteraceae. Alguns dos trabalhos pioneiros nesse campo merecem ser revistos, uma vez que as classificações botânicas sofreram modificações desde então ${ }^{1-4}$. Recentemente, com o aumento do número de metabólitos secundários isolados da família, apareceram alguns artigos tentando utilizar esses dados em estudos quimiotaxonômicos ${ }^{5-7}$. Grandes revisões sobre a presença de substâncias em Asteraceae também foram publicadas e esse acervo foi usado como parte do banco de dados ${ }^{7-9}$. Quimicamente a família é conhecida por produzir principalmente poliacetilenos, sesquiterpenóides, diterpenóides, triterpenóides, flavonóides, cumarinas, benzofuranos e benzopiranos. Outros estudos filogéneticos com base na química macromolecular mudaram bastante a concepção sobre a evolução da família ${ }^{10-12}$. A utilização dos índices de avanço evolutivo baseados em micromoléculas tem sido objeto de estudo por Gottlieb et al. ${ }^{13-15}$. Esse grupo criou uma metodologia inovadora para correlacionar o grau de oxidação de metabólitos secundários e as transformações biogenéticas de seus esqueletos micromoleculares. Os chamados 'diagramas de avanço evolutivo' são adequados para representar muito bem o estágio evolutivo atual dos taxa em vários níveis hierárquicos. O presente trabalho mostra como correlacionar dados oriundos de várias classes químicas, baseado em 10370 ocorrências de micromoléculas, em um único diagrama tridimensional e o compara com a subdivisão da família Asteraceae feita por Wagenitz ${ }^{4}$.

\section{MÉTODOS}

Grande parte dos trabalhos envolvendo oxidação de metabólitos secundários de plantas baseia-se no cálculo do valor do estado de oxidação de cada átomo de carbono da molécula ${ }^{16}$, que somados fornecem o seu grau de oxidação (NOX). As médias dos NOX de todas as ocorrências de uma classe química, em um taxon, representam o AEO (Avanço Evolutivo em Relação a Oxidação) desse taxon, para um determinado tipo de metabólito ${ }^{13}$. Geralmente costuma-se dividir o parâmetro 'NOX' pelo número de átomos de carbonos de cada molécula resultando o parâmetro índice de oxidação (IO). Nem sempre essa divisão é estatisticamente relevante, como no caso

E-mail: vdpemere@quim.iq.usp.br de milhares de sesquiterpenos da nossa base de dados, onde há apenas algumas dezenas de substâncias com 14 átomos de carbono. O uso em conjunto dos índices quimiotaxonômicos para diferentes classes químicas impossibilita a comparação dos números de oxidação ${ }^{13}$. Esses fatos estimularam uma revisão e adaptação da metodologia descrita na literatura ${ }^{13}$. Foi elaborado um novo parâmetro denominado 'passo oxidativo'. Esse parâmetro é obtido subtraindo-se o NOX da substância em questão do NOX do precursor biogenético comum para todos os representantes da classe química e dividindo o resultado por 2. Cada passo oxidativo representa uma insaturação ou uma ligação carbono-heteroátomo presente na dada substância quando comparada ao precursor. Cada diferença de dois pontos no NOX é considerada um passo oxidativo (PO) (Figura 1).

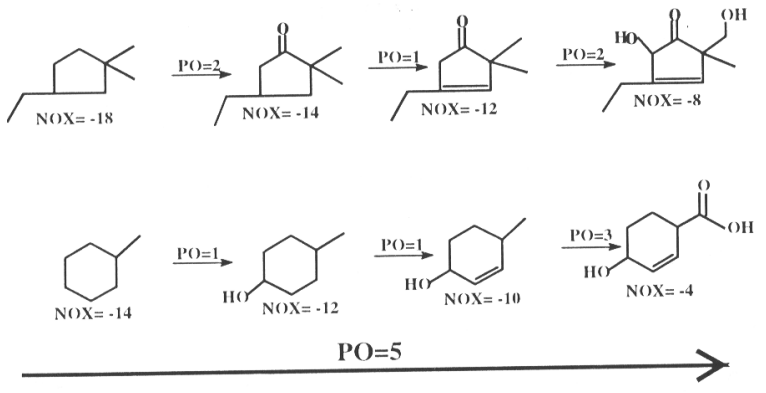

Figura 1. Obtenção dos passos oxidativos (PO) e dos números de oxidação (NOX) para substâncias hipotéticas.

A figura 2 mostra exemplos de cálculos dos passos oxidativos para terpenóides com os respectivos precursores 1, 2, 3 e 4 que têm números de oxidação $-18,-24,-32$ e -50 respectivamente. As substâncias $1 \mathrm{a}, 2 \mathrm{a}, 3 \mathrm{a}$ e $4 \mathrm{a}$ têm números de oxidação e passos oxidativos iguais a $-2(\mathrm{PO}=8),-2(\mathrm{PO})=11,-16(\mathrm{PO})=8 \mathrm{e}$ $-34(\mathrm{PO})=8)$ respectivamente. Isso significa que 2 a utiliza mais "passos oxidativos" do que 1a para chegar até a um idêntico valor de NOX. Com relação as substâncias 3a e 4a, a primeira tem o número de oxidação bem maior que a segunda, embora ambas tenham gasto o mesmo número de etapas oxidativas para chegar ao estágio atual, ou seja, têm os valores de PO idênticos.

Para a obtenção de índices ao nível de tribo, os resultados são bastante confiáveis devido ao grande número de substâncias isoladas (Tabela 1). De maneira análoga ao método usado 


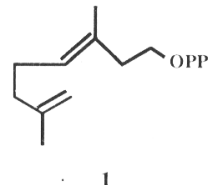

NOX $=-18$<smiles>CC1=CCCCC(C)CCC(C)=CCC1</smiles>

$\mathrm{NOX}=-\mathbf{2 4}$<smiles>CC(C)=CCCC1=CCCC(C)=C1O</smiles>

$\mathrm{NOX}=-32$

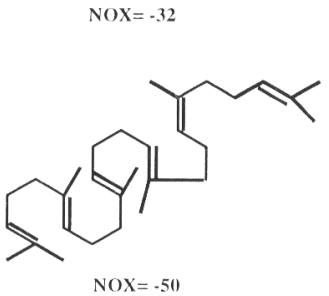

Figura 2. Obtenção dos passos oxidativos (PO) e dos números de oxidação NOX) para terpenóides.

para calcular a oxidação de moléculas orgânicas (NOX), cada mudança no esqueleto (formação ou quebra de uma ligação carbono-carbono), contada a partir do precursor de cada classe (13), é considerada como um estágio de especialização de esqueleto (PE). As médias de PO e PE para um dado taxon como ordem, família, tribo, subtribo e gênero são chamadas de AEPO e AEPE, respectivamente (Tabela 2).

Foi utilizada a classificação de Wagenitz que divide a família Asteraceae em duas subfamílias: Cichorioideae, com a tribo Lactuceae e Asteroideae, subdividida em 2 grupos (GI) e (GII). As tribos adotadas neste estudo são: Anthemideae (ANT, GII), Astereae (AST, GII), Arctoteae (ARC, GI), Calenduleae (CAL, GII), Cynareae (CYN, GI), Eupatorieae (EUP, GII), Heliantheae (incluindo Helenieae) (HLT, GII), Inuleae (INU, GII), Lactuceae (LAC), Liabeae (LIA, GI), Mutisieae (MUT, GI), Senecioneae (SEN, GII), Vernonieae (VER, GI).
Tabela 1. Ocorrências de metabólitos secundários em Asteraceae.

\begin{tabular}{lrrrrr}
\hline \multicolumn{1}{c}{ TRIBO } & LACT & DITE & TRIT & FLAV CUMA \\
\hline LACTUCEAE & 108 & - & 19 & 16 & 56 \\
ARCTOTEAE-GI & 23 & 21 & 35 & - & - \\
CYNAREAE-GI & 253 & 4 & 27 & 80 & 8 \\
LIABEAE-GI & 70 & - & 9 & - & - \\
MUTISIEAE-GI & 84 & 18 & 60 & 13 & 62 \\
VERNONIEAE-GI & 472 & 16 & 177 & 238 & 50 \\
ANTHEMIDEAE-GII & 825 & 9 & 30 & 195 & 168 \\
ASTEREAE-GII & 13 & 1020 & 65 & 109 & 151 \\
CALENDULEAE-GII & - & 94 & 11 & - & - \\
EUPATORIEAE-GII & 445 & 654 & 90 & 180 & 33 \\
HELIANTHEAE-GII & 1645 & 1096 & 65 & 289 & 17 \\
INULEAE-GII & 419 & 406 & 56 & 109 & 24 \\
SENECIONEAE-GII & 104 & 58 & 46 & 22 & 3 \\
\hline
\end{tabular}

Foram estudadas cinco classes de metabólitos secundários isolados de Asteraceae, com uma base de dados contendo 4.461 ocorrências de sesquiterpenóides lactonizados, 3.396 ocorrências de diterpenóides, 690 ocorrências de triterpenóides, 1251 ocorrências de flavonóides ${ }^{17}, 18$ e 572 ocorrências de cumarinas ${ }^{19}$ perfazendo um total de 10370 ocorrências. Cada ocorrência corresponde a um registro de substância no banco de dados, que contém também a tribo, o gênero e a espécie de onde a substância foi isolada, além dos parâmetros PO e PE utilizados neste estudo.

\section{RESULTADOS E DISCUSSÃO}

A tabela 1 mostra os números de ocorrência das 5 classes de metabólitos secundários para cada tribo de Asteraceae arranjadas segundo a classificação de Wagenitz. A tabela 2 mostra os parâmetros evolutivos para as tribos de Asteraceae elaborados com base nos passos oxidativos e nos estágios de especialização de esqueletos para as classes de metabólitos secundários selecionadas. Os gráficos obtidos usando esses parâmetros são mostrados nas figuras 3-5, onde correlacionou-se os valores de AEPO e AEPE para cada classe de terpenóide separadamente. A figura 3 foi elaborada com os dados de lactonas sesquiterpênicas isoladas de Asteraceae. Os parâmetros estatísticos R (Coeficiente de Regressão linear) e $\mathrm{F}$ (Teste de Fischer) foram calculados usando as equações descritas na literatura ${ }^{20}$ e utilizando o programa Stat for Windows ${ }^{21}$. Observa-se para esta classe de produtos naturais que as substâncias pertencentes a esqueletos mais especializados são menos oxidadas. A correlação encontrada entre os valores de

Tabela 2. Parâmetros evolutivos para as tribos de Asteraceae.

\begin{tabular}{|c|c|c|c|c|c|c|c|c|c|}
\hline TRIBO & $\mathrm{AEPO}_{\mathrm{TRI}}$ & AEPO DIT & $\mathrm{AEPO}_{\text {LAC }}$ & AEPE TRI & AEPE DIT & AEPE ${ }_{\text {LAC }}$ & AEPO CUM & AEPO FL-A & $\mathrm{AEPO}_{\text {FL-B }}$ \\
\hline LAC & 0,95 & - & 6,66 & 6,63 & - & 1,87 & 1,87 & 0,90 & 2,79 \\
\hline GI-ARC & 1,06 & 3,67 & 6,48 & 6,80 & 6,17 & 1,74 & - & - & - \\
\hline GI-CYN & 0,78 & 0,25 & 6,64 & 5,52 & 4,75 & 1,83 & 1,75 & 1,56 & 0,99 \\
\hline GI-LIA & 1,44 & - & 6,19 & 6,22 & - & 1,36 & - & - & - \\
\hline GI-MUT & 0,85 & 1,67 & 6,58 & 6,34 & 2,90 & 1,67 & $-0,21$ & 0,84 & 0 \\
\hline GI-VER & 0,88 & 0,64 & 8,70 & 7,18 & 2,90 & 1,38 & $-1,59$ & 0,96 & 2,43 \\
\hline GII-ANT & 0,70 & 0,75 & 6,21 & 4,77 & 3,25 & 1,72 & 2,08 & 1,38 & 1,44 \\
\hline GII-AST & 2,19 & 1,93 & 5,77 & 8,23 & 3,06 & 2,15 & 1,09 & 0,84 & 0,81 \\
\hline GII-CAL & 3,09 & 0,68 & - & 8,91 & 2,98 & - & - & - & - \\
\hline GII-EUP & 0,79 & 1,15 & 7,09 & 6,27 & 3,23 & 1,51 & 0,52 & 0,90 & 0,99 \\
\hline GII-HLT & 2,09 & 1,82 & 7,09 & 7,14 & 5,04 & 2,03 & 1,26 & 1,17 & 0,09 \\
\hline GII-INU & 1,64 & 1,07 & 5,56 & 5,89 & 3,38 & 2,24 & 1,66 & 1,50 & 0,27 \\
\hline GII-SEN & 1,72 & 0,44 & 5,52 & 7,85 & 4,00 & 3,92 & 0,14 & 1,44 & 1,44 \\
\hline
\end{tabular}


AEPO e AEPE para lactonas sesquiterpênicas foi: $\mathrm{R}=0,55$. Para os diterpenos ocorre o fenômeno inverso, ou seja, as mudanças biogenéticas que resultam em estágios de especialização de esqueleto ocorrem paralelamente com o aumento no AEPO (Figura 4), com $R=0,78$. Para os triterpenos (Figura 5) o fenômeno é análogo aos diterpenos. O AEPO aumenta paralelamente com o AEPE $(R=0,78)$. Os três valores de AEPO podem ser vistos em um gráfico tridimensional (Figura 6), onde se nota uma separação de algumas tribos de Asteroideae segundo os subgrupos I e II propostos por Wagenitz ${ }^{4}$. Inuleae, Senecioneae, Heliantheae e Asterae formam um ramo distinto. Calenduleae está nitidamente separada. Anthemideae e Eupatorieae têm posições mais deslocadas para as tribos do grupo I de Asteroideae. A tentativa de achar uma correlação múltipla com os três valores de AEPO de terpenóides resultou em um baixo valor de correlação $(\mathrm{R}=0,44)$. A utilização de apenas três classes de metabólitos para essa correlação pode ser a razão desse baixo valor de $\mathrm{R}$.

Os três valores de AEPE para terpenóides também foram

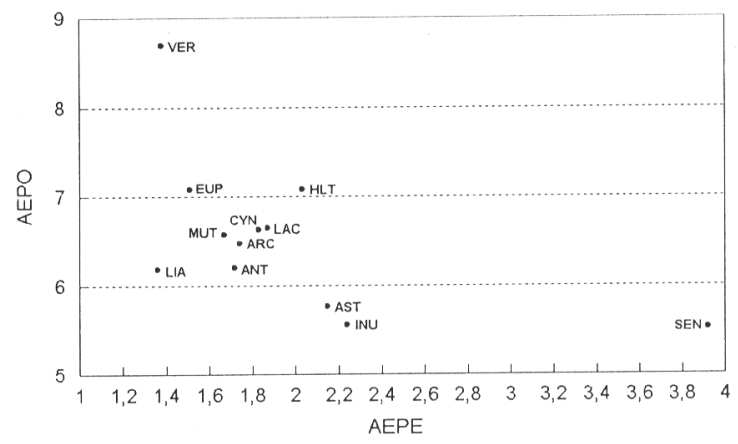

Figura 3. Correlação dos valores de AEPO e AEPE, para tribos de Asteraceae, baseada em lactonas sesquiterpênicas.

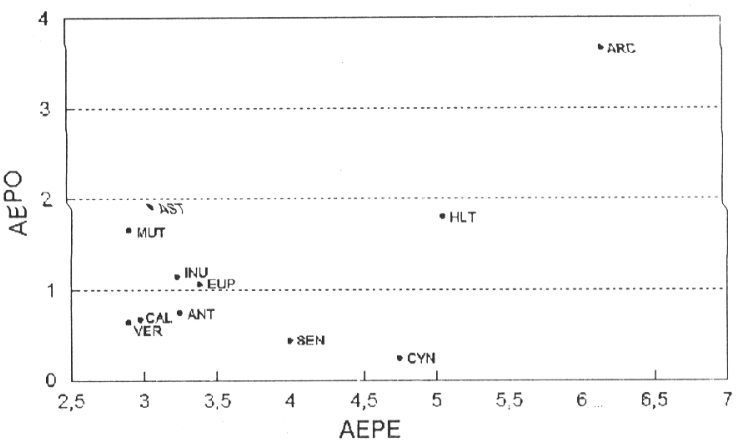

Figura 4. Correlação dos valores AEPO e AEPE, para tribos de Asteraceae, baseada em diterpenóides.

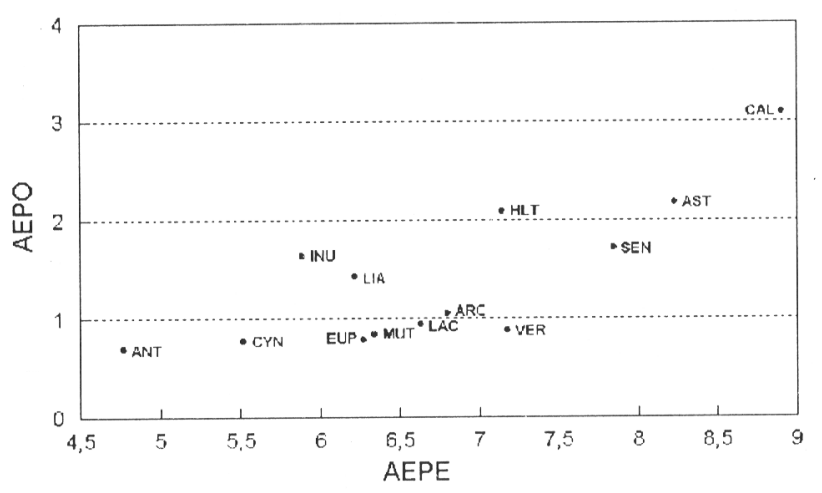

Figura 5. Correlacão dos valores de AEPO e AEPE, para tribos de Asteraceae, baseada em triterpeneóides. utilizados na construção de um diagrama tridimensional (Figura 7). Nesse diagrama Eupatorieae, Anthemideae e Inuleae são as tribos que mais se distanciam de Asteroideae (Grupo II). A correlação múltipla para todos os valores de AEPE também forneceu um baixo valor de correlação $(R=0,44)$.

A análise das figuras 6 e 7 é interessante também para realçar a grande diferença entre os índices de Calenduleae e das outras tribos de Asteroideae (Grupo II).

As correlações de AEPO somente para os três parâmetros vistos anteriormente podem ser melhoradas ao acrescentarmos os valores obtidos para cumarinas e para flavonóides. Os valores de AEPO para essa última classe de metabólitos, são calculados separadamente para a parte derivada da via do ácido acético e para a parte derivada da via do ácido chiquimico (FL-B). As variações dos valores de oxidação das 5 classes de metabólitos para as tribos de Asteraceae e a preponderância da oxidação de lactonas sesquiterpênicas sobre os demais metabólitos podem ser visualizadas graficamente na figura 8 .

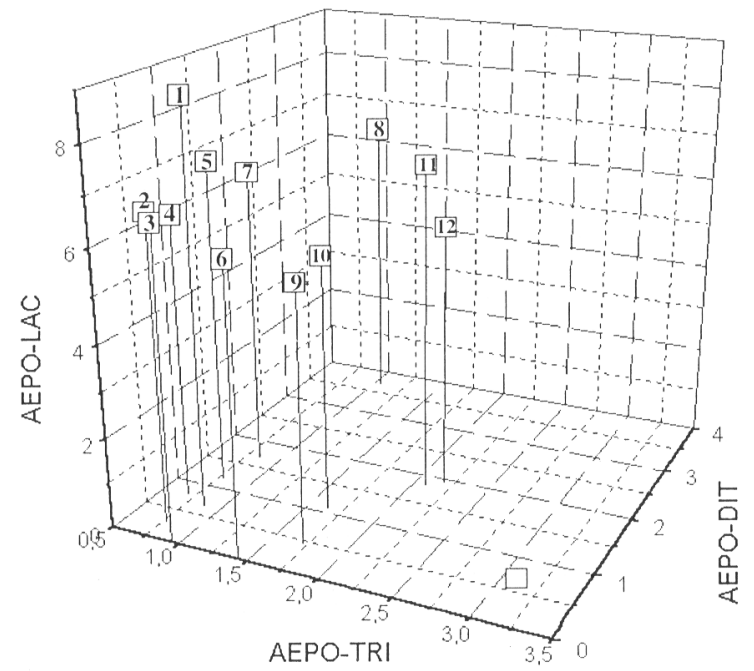

Figura 6. Diagrama de afinidades com base nos índices de avanço evolutivo, em relação à oxidação de três classes de terpenóides, para tribos de Asteraceae. $1=V E R, 2=C Y N, 3=L A C, 4=A N T, 5=E U P$, $6=L I A, 7=M U T, 8=A R C, 9=S E N, 10=I N U, 11=H L T, 12=A S T$.

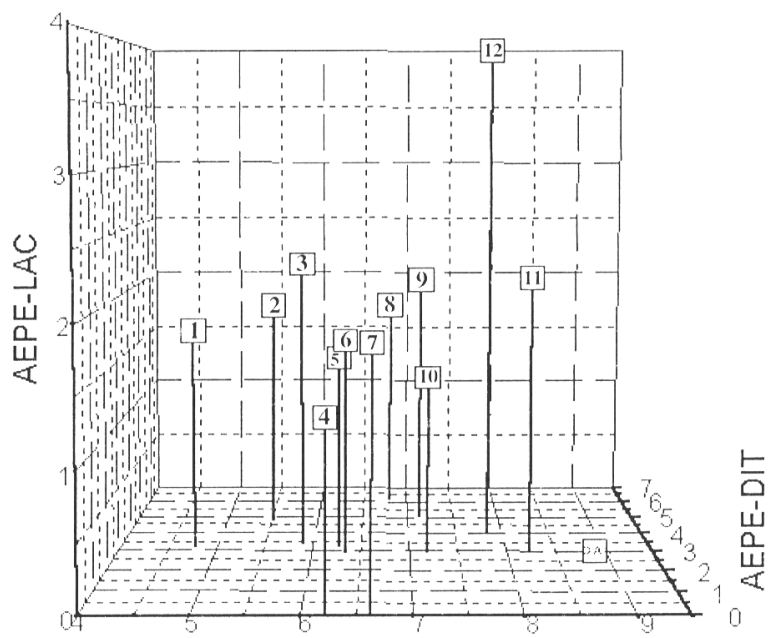

AEPE-TRI

Figura 7. Diagrama de afinidades com base nos índices de avanço evolutivo, em relação à especialização de esqueletos, de três classes de terpenóides (lactonas sesquiterpênicas, diterpenos e triterpenos), para tribos de Asteraceae. $l=A N T, 2=C Y N, 3=I N U, 4=L I A, 5=E U P$, $6=M U T, 7=L A C, 8=A R C, 9=H L T, 10=V E R, 11=A S T, 12=S E N$. 


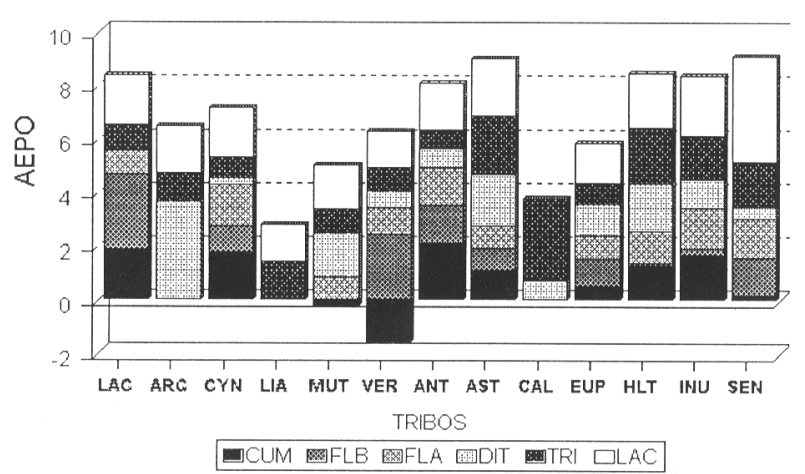

Figura 8. Variação dos valores de avanço evolutivo de metabólitos secundários para tribos de Asteraceae.

Abaixo são fornecidas as equações encontradas para os seis parâmetros oxidativos mostrando para cada equação o coeficiente de regressão linear e teste de Fischer.

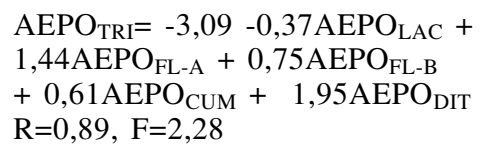

$\mathrm{AEPO}_{\mathrm{CUM}}=-5,46-0,60 \mathrm{AEPO}_{\mathrm{TRI}}-0,36 \mathrm{AEPO}_{\mathrm{LAC}}$ $+1,45 \mathrm{AEPO}_{\mathrm{FL}-\mathrm{A}}+0,47 \mathrm{AEPO}_{\mathrm{FL}-\mathrm{B}}+1,60 \mathrm{AEPO}_{\mathrm{DIT}}$ $\mathrm{R}=0,88, \mathrm{~F}=2,17$

$\mathrm{AEPO}_{\mathrm{DIT}}=2,12+0,37 \mathrm{AEPO}_{\mathrm{TRI}}+$ $0,12 \mathrm{AEPO}_{\mathrm{LAC}}+-0,75 \mathrm{AEPO}_{\mathrm{FL}-\mathrm{A}}+$ $-0,41 \mathrm{AEPO}_{\mathrm{FL}-\mathrm{B}}+0,29 \mathrm{AEPO}_{\mathrm{CUM}}$ $\mathrm{R}=0,98, \mathrm{~F}=14,65$

A figura 9 mostra os valores de AEPO calculados versus observados diterpenos usando a equação [6] acima. A maior diferença encontrada entre AEPO calc. e obs. foi de 0,277 , ou seja, um quarto de um passo oxidativo.

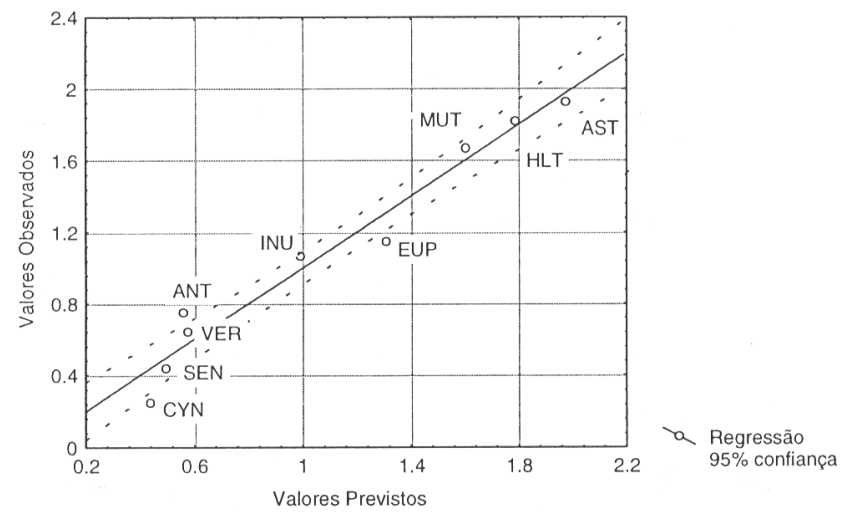

Figura 9. Exemplo de AEPO previsto versus AEPO calculado, para tribos de Asteraceae.
Em estudos anteriores demonstrou-se que durante a evolução de micromoléculas a oxidação geralmente acompanha a especialização de esqueletos ${ }^{13}$. Posteriormente, foi verificada que, para determinado taxon, essa tendência, pode ser vista em um certo nível hierárquico e que o fenômeno inverso pode ocorrer em um outro nível hierárquico ${ }^{14}$. A análise das Figuras 3, 4 e 5 permite avaliar que ambos os fatos, especialização de esqueletos com aumento de oxidação, ou com diminuição da oxidação, podem ocorrem em um mesmo nível hierárquico, dependendo da classe de metabólito analisada.

Esse fato pode ser observado graficamente ou pela inspeção das estruturas das substâncias comumente isoladas de tribos da família ${ }^{7}$. No caso de lactonas sesquiterpênicas a oxidação é preponderante nos germacranolídeos (Figura 2, substância 2a), que é o tipo mais comum. Existe também grande variação de reações oxidativas nos derivados de germacranolídeos, que podem ter várias conformações suscetíveis de serem oxidadas. Esses derivados, apesar de não sofrerem formação ou quebra de ligação carbono-carbono, são considerados na literatura ${ }^{7}$ como esqueletos diferentes. Para os outros sesquiterpenos lactonizados as transformações de esqueleto não envolvem necessariamente mudanças oxidativas e resultam de migração de grupos metila e formação de novas ligações C-C.

\section{CONCLUSÕES}

Os diagramas de afinidade evolutiva fornecem agrupamentos e algum tipo de correlação entre dois parâmetros. Para as três classes de terpenóides de Asteraceae as correlações obtidas entre AEPO e AEPE são aceitáveis para lactonas sesquiterpênicas e, por se tratar de fenômenos biológicos, muito boas para diterpenóides e triterpenóides. Os diagramas tridimensionais são bastante úteis para mostrar o parentesco real entre tribos com base em três parâmetros.

As correlações múltiplas entre índices de oxidação das 5 classes de metabólitos sugerem que deve haver um equilíbrio bioquímico na oxidação das micromoléculas e na especialização dos esqueletos dessas substâncias. Os altos valores de $\mathrm{R}$ e os bons resultados encontrados entre valores previstos e observados justificam a utilização dessa nova abordagem para avaliar o posicionamento filogenético do taxon.

Com a ajuda dos diagramas pode-se verificar, por exemplo, que apesar de Wagenitz classificar a tribo Calenduleae como Asteroideae (Grupo II), ou Bremer colocá-la em seus diagramas filogenéticos perto das outras tribos de Asteroideae, que essa tribo não acompanhou a evolução química da família quanto a produção de micromoléculas e quando as produz em pequenas quantidades os parâmetros evolutivos calculados também mostram sua posição diferenciada.

Vernonieae e Liabeae são consideradas tribos muito próximas com base em dados macromoleculares, porém nesse trabalho fenômeno inverso foi verificado.

A metodologia descrita aqui, permite descrever com clareza, usando quantas classes de marcadores químicos estiverem disponíveis, o parentesco real de tribos, ou de outros níveis hierárquicos. Um entendimento global da evolução da família depende de uma interpretação conjunta de dados oriundos de macro e micromoléculas, dados botânicos e de outras abordagens.

\section{AGRADECIMENTOS}

Ao CNPq e à FAPESP pelas bolsas concedidas.

\section{REFERÊNCIA}

1. Bentham, G.; J. Linn. Soc. Bot. 1873, 13, 335.

2. Carlquist, S.; Aliso 1976, $8,465$.

3. Cronquist, A.; Brittonia 1977, 29, 137.

4. Wagenitz, G.; Pl. Syst. Evol. 1976, 125, 29. 
5. Emerenciano, V. P.; Bonfanti, M. R.; Ferreira, Z. S.; Kaplan, M. A. C.; Gottlieb, O. R.; Biochem. Sys. Ecol. 1987, 14, 585.

6. Emerenciano, V. P.; Ferreira, Z. S.; Kaplan, M. A. C.; Gottlieb, O. R.; Phytochemistry 1987, 26, 3103.

7. Seamann, F. C.; Bot. Rev. 1982, 48, 125.

8. Seamann, F. C.; Bohlmann, F.; Zdero, C.; Mabry, T. J.; Diterpenes of Flowering Plants - Compositae(Asteraceae). Springer-Verlag, New York, 1990.

9. Bohlmann, F. e Zdero, C.; Plant Syst. Evol. 1990, 171, 1.

10. Bremer, K.; Cladistics 1987, 3, 210.

11. Bremer, K.; Asteraceae. Cladistics and Classification, Timber Press, Portland, 1994.

12. Jansen, R. K.; Holsingeer, K. E.; Michaels, H. J.; Palmer, J. D.; Evolution 1990, 44, 2089.

13. Gottlieb, O. R.; Micromolecular Evolution Systematics and Ecology. Springer-Verlag, Berlin, 1982.
14. Gottlieb, O. R.; Phytochemistry 1989, $28,2545$.

15. Gottlieb, O. R.; Phytochemistry 1990, 29, 1715.

16. Hendrickson, J. B.; Cram, D. J.; Hammond, G. S.; Organic Chemistry. $3^{\mathrm{a}}$ ed. McGraw-Hill, New York, 1970.

17. Harborne, J. H.; Mabry, T. J.; Mabry, H. (eds) The Flavonoids. Chapman and Hall, Londres, 1975.

18. Harborne, J. H.; Mabry, T. J. (eds.) The Flavonoids Advances in Research. Chapman e Hall, Londres, 1982.

19. Robert, D. H.; Mendez, J.; Brown. The Natural Coumarins. Chemistry and Biochemistry, John Wiley \& Sons, 1982.

20. Costa Neto, P. L. D.; Estatística. Editora Edgard Blucher Ltda, São Paulo, 1977.

21. Norusis, M. J.; SPSS, Statistical Package. Chicago, Il., USA, 1986. 